(c) 2010 IEEE. Personal use of this material is permitted. Permission from IEEE must be obtained for all other uses, in any current or future media, including reprinting/republishing this material for advertising or promotional purposes, creating new collective works, for resale or redistribution to servers or lists, or reuse of any copyrighted component of this work in other works. 


\section{Analysis on Intra-aneurysmal Flow Influence By Stenting}

\author{
Kelvin K. L. Wong, Pongpat Thavornpattanapong and Jiyuan Tu \\ School of Aerospace, Mechanical \& Manufacturing Engineering, \\ and Health Innovations Research Institute (HIRi), \\ RMIT University, Australia \\ \{k.wong,pongpat.thavornpattanapong,jiyuan.tu\}@rmit.edu.au
}

\author{
Zhonghua Sun \\ Discipline of Medical Imaging, \\ Department of Imaging and Applied Physics, \\ Curtin University of Technology, Australia \\ z.sun@curtin.edu.au
}

\begin{abstract}
Using numerical simulation, the evolution of vortices in an aneurysm can be tracked. We examined large-scale swirling of blood within a significantly dilated aneurysm and quantified the vorticity and shear strain rate. Based on these fluid mechanical parameters, we are able to identify the difference in flow effects between the untreated and stented aneurysmal arteries. This study demosntrates that the large-scale vortex and blood shear strain rate within an aneurysm sac reduces after stenting.
\end{abstract}

Index Terms-Flow analysis;Computational fluid dynamics;Aneurysm; Vorticity;Shear strain rate.

\section{INTRODUCTION}

Aneurysm is a blood filled dilation that is caused by atherosclerotic disease of blood vessel walls and is common near the branches of intracranial arteries. With aging, aneurysms increase in size resulting in the rupture and initiation of bleeding within the brain. This causes a medical condition known as hemorrhagic stroke that can result in death or disability. Hemorrhagic stroke is known to be one of the common causes of death by cardiovascular disease and affects $15 \%$ of stroke patients in the world's population [1]. The affected arterial region is generally treated using angioplasty with stents or by open surgery that consist of clipping whereby a clip is inserted across the aneurysm in order to prevent blood from entering the aneurysmal bulge and aggravating its dilation. The causes of this cardiovascular disease are diabetes, obesity, alcoholism, tobacco use and a copper deficiency [12]. Patients who die from a medical condition have copper deficiency of approximately quarter quantity of a normal person. It might be worthwhile noting that tobacco and alcohol intake diminishes the copper content within the body, resulting in the progression of aneurismal growth as portrayed by [11]. To investigate the development of aneurysm, Computer Fluid Dynamic (CFD) techniques are employed to quantify blood flow through arteries based on idealistic geometries and realistic geometries defined from medical imagining techniques such as Magnetic Resonance Imaging and Computed Tomography. Mechanical parameters such as Wall Shear Stress (WSS), wall pressure and velocity can quantify the nature of intra-aneurismal flow [12]. Vortex regions that portray the spiraling effect can then be analyzed in the affected arteries in order to determine initiation and progression of an aneurysm leading to its final rupture.

The pioneer proposal and development of prosthetic devices that maintain the luminal integrity of diseased blood vessels took place in 1964, which leads to the first implantation of stents into humans in 1986. Since then, numerous innovations and modifications are performed to improve its safety and functionality [9]. The presence of a non-biocompatible device inside an artery leads to inevitable inflammation and influences the fluid dynamic behavior in the regions next to the arterial wall. Parts of the stent struts protruding into the lumen may induce the formation of vortices and stagnation zones which affect wall shear stress. These effects depend on parameters such as the stent configuration, its global length, the delivery system, the struts dimension, shape, and spacing [10]. Stents that are poorly matched to patient-specific vessels may pose vascular complications [8], [2], [5]. In cases of serious conditions, subacute thrombosis due to wrong use of stents may result [13]. In this project, cardiac flow analysis will be able to reveal insight into parameters that have the most significant effect on flow behavior due to the implementation of stent and risk of re-stenosis.

Based on numerical simulation, we can study the change in intra-aneurysmal flow field for a diseased artery before and after stenting. Vorticity mapping can be performed by processing the simulated velocity field and used to analyse the large-scale vortex in the aneurysm sac. To quantify the complex aneurysmal flow, statistical data is compiled from each well-defined vortex. Information from these component statistics may be able to present the flow characteristics and relate to the stress and strain due to swirling of the blood within an aneurysm. This paper presents the assessment of largescale vortices by using computational fluid dynamics to simulate the evolution of this aneurysmal flow for both the non-stented and stented arterial configuration. 


\section{MAterials \& MethodS}

\section{A. Computational Fluid Dynamics}

The governing equations for viscous, incompressible fluid flow can be written using Navier-Stokes equations and mass continuity equation as

$$
\begin{aligned}
\rho_{L} \frac{d V}{d t}= & \rho_{L} g+\mu \nabla^{2} V-\nabla P, \\
& \frac{\partial \rho_{L}}{\partial t}+(\nabla \cdot V) \rho_{L}=0,
\end{aligned}
$$

where $V$ is the fluid velocity vector. $\mu$ is the viscosity, $g$ is the gravitational acceleration, $\rho_{L}$ is the density, and $P$ is the dynamic pressure of the fluid.

Vortices that exist in a stented aneurysm may be driven by orifice or aneurysm flow [3]. Numerical simulation of flow in stented aneurysms is performed in various studies. In particular, the use of Lattice Boltzmann approach is heavily implemented [3], [13], [4]. Numerical simulation based on untreated and stented aneurysmal arteries may be performed to demonstrate their difference in terms of blood flow. The modelling of circular stent struts at the location of the aneurysm is used for the stented artery.

\section{STENTING CONFIGURATION}

Non-stented Artery

Fig. 1: Schematic of non-stented and stented aneurysmal artery.

\section{B. Post-processing of Simulated Flow}

Vorticity is quantified based on the fluid velocity gradients of the fluid at a point of interest [7]. The circulation is the line integral of the tangential velocity along a circuit (in a counter-clockwise fashion) enclosing that point of fluid rotation. The vorticity $\omega$ is then equal to the circulation divided by the area enclosed by this circuit.

In general, two-dimensional vorticity is expressed mathematically as

$$
\omega=\left(\frac{\partial V_{y}}{\partial x}-\frac{\partial V_{x}}{\partial y}\right)_{i, j} .
$$

In our convention, positive values signify counterclockwise $(\mathrm{CCW})$ circulation, whereas negative values represent clockwise (CW) rotation of the fluid. Therefore, the magnitudes of these values give an indication of the rate of rotation and its polarity signifies the direction of the rotation.

In a fluid flow that is incompressible and under a steady flow field, the shear rate of strain of a material fluid element is defined as the rate of decrease of the angle formed by two mutually perpendicular lines on the element. As such, the shear strain rate $\dot{\gamma}$ is defined as a function of $\tau$ and is proportional to the velocity gradient in the perpendicular direction of the flow:

$$
\dot{\gamma}=f(\tau)=-\frac{d u}{d y} .
$$

In general, the shear strain rate can be defined as a function of $\tau$, and we establish a linear relationship between the shear stress and strain components with the viscosity as its gradient. Mathematically, the model can be expressed as

$$
\tau=\mu \dot{\gamma},
$$

where $\mu$ is the viscosity of the fluid.

\section{EXPERIMENTS}

\section{A. Geometrical Domain}

The computational domain of the aneurysmal model is shown in Fig. 2. The parent vessel and the aneurysmal sac are set at $8 \mathrm{~mm}$ and $5 \mathrm{~mm}$ in diameters respectively. The flow will enter the vessel with no obstacle and swirl within the aneursym. For the stented artery, the aneurysmal flow velocity is reduced due to the stent struts obstructing blood movement. For simplicity, the velocity boundary condition that was applied at the arterial entrance assumes a uniform profile and has a direction that is normal to the surface. The artery is modelled to be long enough so that towards the exit of the vessel, the zero pressure gradient boundary condition along the length of the artery is implemented.

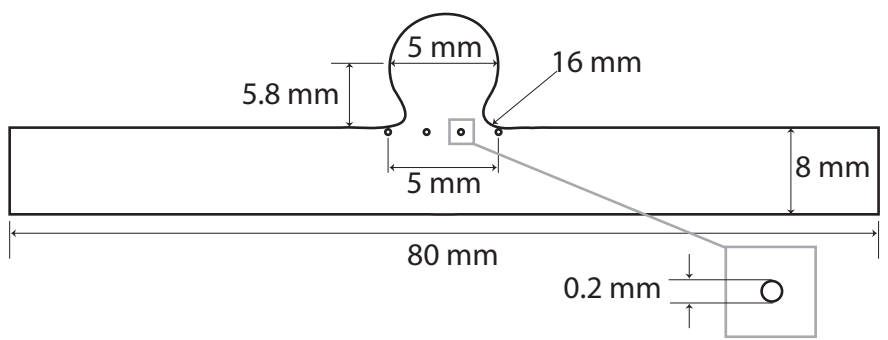

Fig. 2: Dimensions of aneurysm model.

A close-up view of the mesh for this aneurysmal vessel (Fig. 3) shows the variation of tetrahedral cells at the aneurysmal wall and around the stent struts, A domain size of $6 \mathrm{k}$ cells is generated. A mesh independence study has already been carried out to ascertain that the selected domain size is sufficient in resolving the flow gradients. 


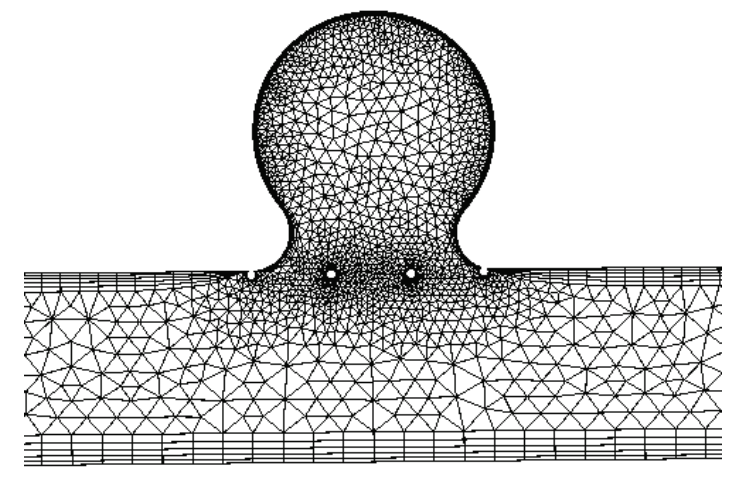

Fig. 3: Computational mesh of aneurysm model.

\section{B. Numerical Simulation}

After the processing of geometrical mesh, the next step in the preprocessing stage of the CFD workflow is to define and subsequently, implement the inputs for the physical models and boundary conditions. Here, we have assumed that flow of blood through the arterial vessel is incompressible, homogeneous, laminar, and Newtonian. The fluid properties are reported in Table I. It may be worthwhile noting that Reynolds number in the cerebral artery typically has a range of 100 to 300 , and is less than 30 within the aneurysm [4]. To model the unsteady nature of the flow field, transient CFD simulation was performed. Rigid wall condition is assumed.

TABLE I: Fluid properties used in numerical simulation

\begin{tabular}{|c|c|c|}
\hline Parameter & Value & Units \\
\hline \hline Dynamic viscosity & $3.5 \times 10^{-3}$ & Pascal \\
\hline Density & 1050 & $\mathrm{kgm}^{-3}$ \\
\hline Entrance velocity & 0.04377 to 8.088 & $\mathrm{cms}^{-1}$ \\
\hline Exit velocity & 0 to 8.33 & $\mathrm{cms}^{-1}$ \\
\hline Pressure & 0 to 100 & \\
\hline
\end{tabular}

Software ANSYS CFX has been utilised for transient computational fluid dynamic simulations. The discretization of Navier Stokes equation is based on coupling both finite-element and finite-volume approaches. The flow in the vessel is simulated from the the earlysystole state to end-diastolic state of the cardiac cycle $T$ based on an idealistic physiological waveform (refer to Fig 4). We simulate the model for one cardiac time cycle $T$ from 0 to 1 . The pressure starts from zero, and the peak pressure and flow rate occurs at $t / T=0.15$. The pressure then reduces to zero again at $t / T=0.3$ and then continues at zero pressure till $t / T=1$. This pressure variation is repeated again in the subsequent cycles.

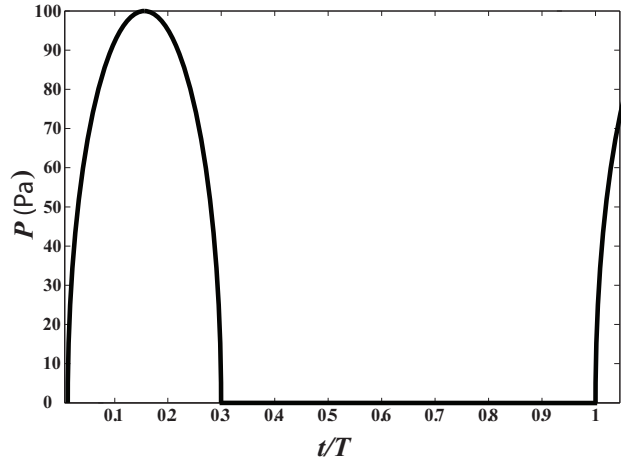

Fig. 4: Plot of pressure versus time.

\section{RESULtS}

Streamline tracing is used to deduce the path of the blood flow. A clearly defined large-scale vortex is present in the aneurysm sac as shown in Fig. 5. The results show that an aneurysmal vortex reduces in strength after stenting.

STENTING CONFIGURATION

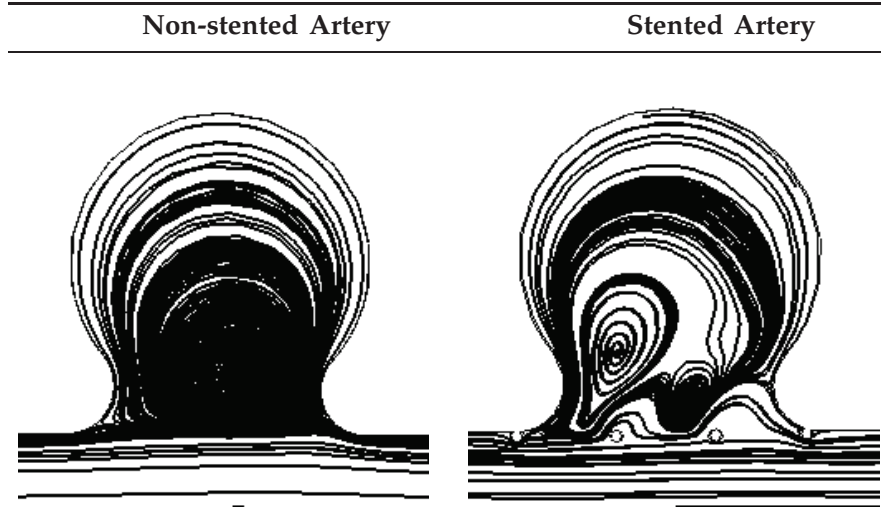

Fig. 5: Streamline tracing of aneurysmal flow.

Fig. 6 presents the counter-clockwise vortices in the aneurysm sac using vorticity and velocity vector fields. In the non-stented artery, a large-scale vortex exist in the aneurysm sac. When stented, we note that the strength of the vortex decreases as demonstrated by their lower vorticity values. We characterise these vortices by quantification of the vorticity $\omega$ and shear strain rate $\dot{\gamma}$ using a monitor point at the centre of the aneurysmal sac (indicated by a crosshair in the flow field plots). The vorticity and shear strain rate fields can give a quantifiable indication of the vortex differences for the untreated and stented aneurysmal artery based on time frame indices $t / T=0.1,0.3,0.6$ and 1.0 . 


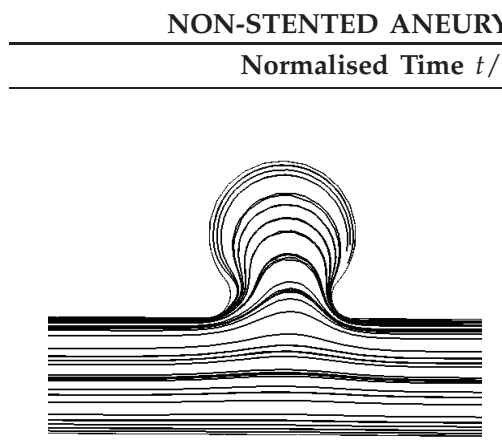

(i) $t / T=0.1, \omega=-7.13 \mathrm{~s}^{-1}, \dot{\gamma}=1.53 \mathrm{~s}^{-1}$

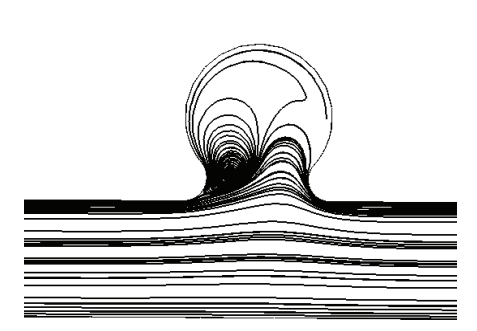

(ii) $t / T=0.3, \omega=-7.13 \mathrm{~s}^{-1}, \dot{\gamma}=4.56 \mathrm{~s}^{-1}$

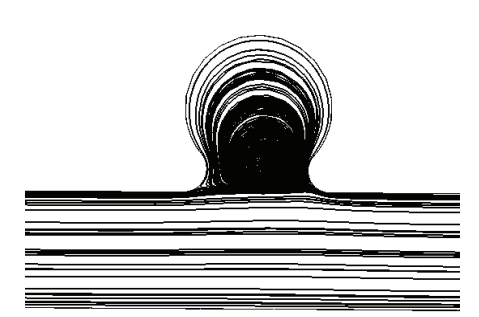

(iii) $t / T=0.6, \omega=-9.44 \mathrm{~s}^{-1}, \dot{\gamma}=6.81 \mathrm{~s}^{-1}$
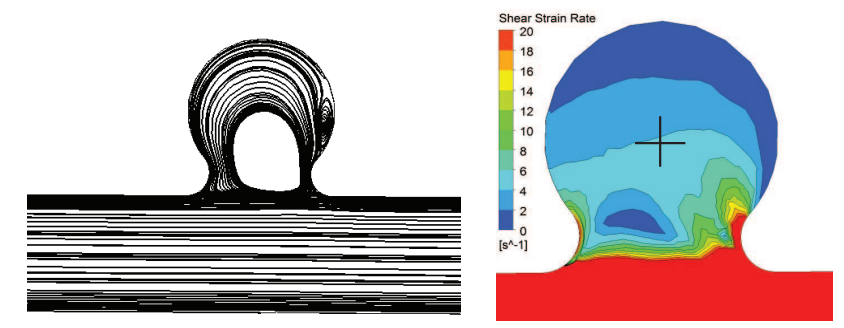

(iv) $t / T=1.0, \omega=-7.44 \mathrm{~s}^{-1}, \dot{\gamma}=3.88 \mathrm{~s}^{-1}$

(a) Non-stented aneurysmal flow analysis for time $t / T=0.1$ to 1.0

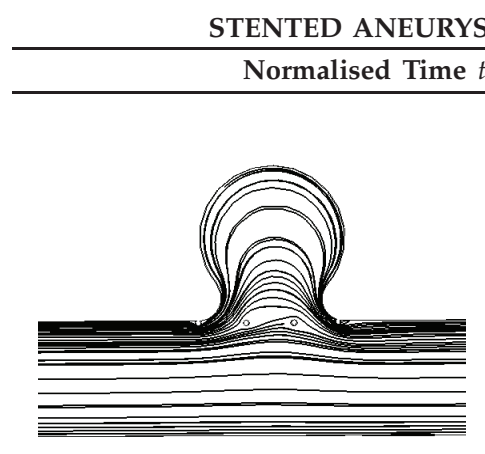

(i) $t / T=0.1, \omega=11.44 \mathrm{~s}^{-1}, \dot{\gamma}=1.72 \mathrm{~s}^{-1}$
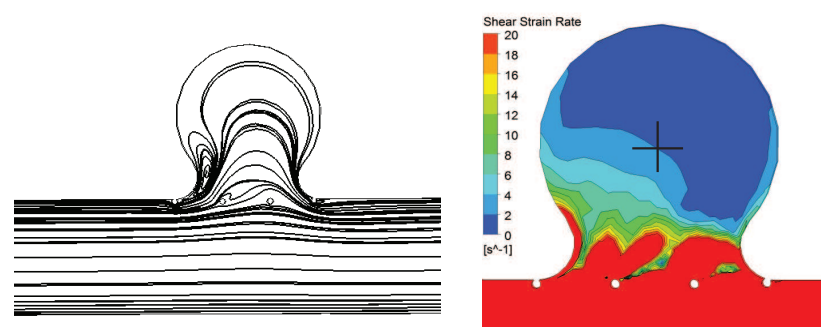

(ii) $t / T=0.3, \omega=11.44 \mathrm{~s}^{-1}, \dot{\gamma}=1.66 \mathrm{~s}^{-1}$
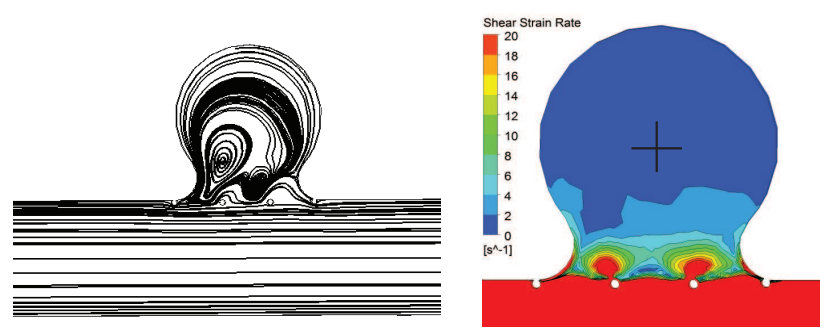

(iii) $t / T=0.6, \omega=18.87 \mathrm{~s}^{-1}, \dot{\gamma}=1.06 \mathrm{~s}^{-1}$
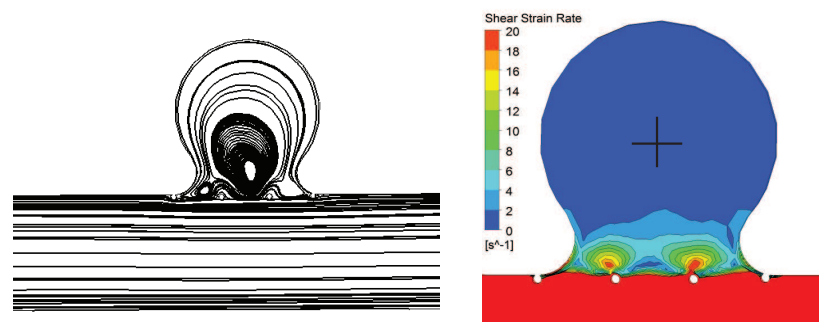

(iv) $t / T=1.0, \omega=12.50 \mathrm{~s}^{-1}, \dot{\gamma}=0.78 \mathrm{~s}^{-1}$

(b) Stented aneurysmal flow analysis for time $t / T=0.1$ to 1.0

Fig. 6: Aneurymal flow analysis based on non-stented and stented arteries. 


\section{DISCUSSION}

Large-scale vortices are identified for four time frames of one cardiac cycle. For the case of the non-stented aneurysmal flow, a vortex of counter-clockwise motion has vorticity $\omega$ that ranges from $=-9.44$ to $-7.59 \mathrm{~s}^{-1}$, while the vorticity for the stented case has $\omega$ with range 11.44 to $18.87 \mathrm{~s}^{-1}$. At the monitor point in the center of the aneurysmal bulge, the shear strain rate for the non-stented condition is almost the same as the stented case initially at $t / T=0.1$ (with $\dot{\gamma}=1.53$ and $1.72 \mathrm{~s}^{-1}$ for untreated and stented cases respectively) but is shown to have a decrease after the stenting from $\dot{\gamma}=4.56$ to $1.655 \mathrm{~s}^{-1}$ for $t / T=0.3$, from $\dot{\gamma}=6.81$ to $1.058 \mathrm{~s}^{-1}$ for $t / T=0.6$, and from $\dot{\gamma}=3.88$ to $0.782 \mathrm{~s}^{-1}$ for $t / T=1.0$.

Based on numerical simulation and flow analysis, we are able to characterise flow fields using vorticity measurement on untreated and stented aneurysmal arteries. The vorticity map enables the visualisation of strength and position of a large-scale vortex in an aneurysmal sac. The results demonstrate that the stenting causes a reduction of velocity, vorticity shear rate. However, lower volume of flow into the sac increases the viscosity of blood in the aneurysm [4]. Reduced vorticity in the sac also means a lower wall shear stress. Flow stagnancy and the induction of thrombosis increases. All these conditions if increased excessively will aggravate aneurysm rupture [6]. Therefore, the type of stents deployed has to be of sufficient porosity to minimise aneurysmal rupture [4]. The study of fluid mechanical properties in nonstented and stented aneurysmal flow can enable medical experts to evaluate the effectiveness of stent designs and their corresponding porosities in prevention of aneurysm dilation leading to rupture.

\section{CONCLUSION}

In this paper, we present a simulated model to show stenting inside the diseased artery at the entrance of the aneurysm, and demonstrate that the interference with blood flow is different from the situation before stent treatment. The reason to insert stents inside the aneurysm is to embolize it or exclude it from systemic blood circulation, so that the aneurysm will shrink gradually and eventually becomes smaller. It is true that this may induce stagnation and possible thrombosis formation after stenting; however, as long as the aneurysm does not grow, the chance of being ruptured is low, so the goal of preventing it from rupture is achieved. Aneurysm can be formed on both sides of the artery wall and so for future work, simulation of different types of diseased arterial geometries will be more useful for understanding of the effect of stent treatment.

\section{ACKNOWLEDGMENT}

The authors thank Mr Manisha Dabarera and Mr Mohammad Rasidi Mohammad Rasani for their kind assistance in post-processing the simulation results in this paper. The financial support provided by the Australian Research Council (ARC project ID DP0986183) is gratefully acknowledged.

\section{REFERENCES}

[1] AIHW Board. Australia's Health 2008. Number AUS 99. Australian Institute of Health and Welfare, 2008.

[2] P. C. Gordon, S. P. Friedrich, R. N. Piana, A. D. Kugelmass, G. A. Leidig, C. M. Gibson, D. J. Cohen, J. P. Carrozza, R. E. Kuntz, and D. S. Baim. Is 40directional coronary atherectomy clinically significant? The American Journal of Cardiology, 74(1):26-32, 1994.

[3] M. Hirabayashi, M. Ohta, K. Barath, D. A. Rufenacht, and B. Chopard. Numerical analysis of the flow pattern in stented aneurysms and its relation to velocity reduction and stent efficiency. Mathematics and Computers in Simulation, 72(2-6):128-133, 2006.

[4] Y. H. Kim, X. Xu, and J. S. Lee. The effect of stent porosity and strut shape on saccular aneurysm and its numerical analysis with lattice boltzmann method. Annals of Biomedical Engineering, 38(7):2274-2292, 2010.

[5] R. E. Kuntz and D. S. Baim. Defining coronary restenosis: newer clinical and angiographic paradigms. Circulation, 88:1310-1323, 1993.

[6] T. M. Liou and S. N. Liou. Pulsatile flows in a lateral aneurysm anchored on a stented and curved parent vessel. Experimental Mechanics, 44(3):253-260, 2004.

[7] M. Raffel, C. Willert, and J. Kompenhans. Particle Image Velocimetry. Springer-Verlag, Berlin Heidelberg, Germany, 1998.

[8] C. Rogers and E.R. Edelman. Endovascular stent design dictates experimental restenosis and thrombosis. Circulation, 91:29953001, 1995.

[9] M. E. Silverman, N. G. Kounis, G. N. Kounis, S. N. Kouni, D. P. Taggart, P. W. Serruys, and A. T. L. Ong. Coronary-artery stents. N. Engl. J. Med., 354(19):2076-2078, 2006.

[10] R. Tominaga, H.E. Kambic, H. Emoto, H. Harasaki, C. Sutton, and J. Hollman. Effects of design geometry of intravascular endoprostheses on stenosis rate in normal rabbits. American Heart Journal, 123:2128, 1992.

[11] B. Utter and J. S. Rossmann. Numerical simulation of saccular aneurysm hemodynamics: Influence of morphology on rupture risk. Journal of Biomechanics, 40(12):2716-2722, 2007.

[12] A. Valencia, H. Morales, R. Rivera, E. Bravo, and M. Galvez. Blood flow dynamics in patient-specific cerebral aneurysm models: The relationship between wall shear stress and aneurysm area index. Medical Engineering \& Physics, 30(3):329-340, 2008.

[13] X. Xu and J. S. Lee. Application of the lattice boltzmann method to flow in aneurysm with ring-shaped stent obstacles. Int. J. Num. Meth. Fluids, 59(6):691-710, 2008. 